\title{
Dynamics of Thrombus Formation in Mouse Testicular Surface Vein Visualized by Newly Devised "Vascular Mapping" Method for Live-CLEM Imaging in vivo.
}

\author{
Akira Sawaguchi ${ }^{1}$, Satoshi Nishimura ${ }^{2,3,4}$ \\ 1. Department of Anatomy, Ultrastructural Cell Biology, Faculty of Medicine, University of Miyazaki, \\ Miyazaki, Japan. \\ 2. Department of Cardiovascular Medicine, The University of Tokyo, Tokyo, Japan \\ 3. Translational Systems Biology and Medicine Initiative, The University of Tokyo, Tokyo, Japan \\ 4. Center for Molecular Medicine, Jichi Medical University, Tochigi, Japan
}

One of the goals of biomedical microscopy is to elucidate a functional morphology in vivo, leading to associated pathophysiology and further clinical investigation. The dynamics of thrombus formation has yet to be elucidated to prevent human diseases, such as infarction in the heart and the brain, etc. Most recent progress of in vivo multi-photon laser scanning microscopy (LSM) enables us to visualize experimentally induced vascular damage followed by thrombus formation in blood vessels of mice [1-3]. However, a new reliable method remains to be developed to acquire a transmission electron microscopic (TEM) image of the experimentally formed thrombus in situ, hampered by difficulties in locating and processing the thrombus to be cut into ultrathin sections.

To address this problem, we newly developed a "Vascular Mapping" method for correlative light and electron microscopy (CLEM) of thrombus formation in vivo. Briefly, after anesthesia, Texas-Reddextran was injected to visualize blood flow, and then the mouse testicular surface vein was exposed onto a multi-photon LSM. Following a capture of complex vascular pattern at lower magnification (Fig 1), the focused region was damaged by laser irradiation to obtain sequential images of thrombus formation in situ (Fig 2).

For TEM image preparation, the testis was excised and immersed into half-strength Karnovsky fixative. Then, the focused region was excised referring the captured image of complex vascular "map" (Fig 3), and processed to be embedded into epoxy resin. Next, semi-thin sections ( $3 \mu \mathrm{m}$ in thickness) were sequentially cut tracing upstream of the damaged vein to reach the thrombus (asterisk in Fig 4A). The obtained semi-thin section of thrombus was re-embedded into epoxy resin to be cut into ultrathin sections (70 $\mathrm{nm}$ in thickness) using capsule-supporting ring [4].

As results, the present "vascular mapping" approach succeeded in TEM observation of the thrombus as shown in Fig 4B and 4C, yielding live-CLEM imaging in vivo. The preliminary observation demonstrated a noteworthy attachment of neutrophils (Fig 4B) onto the thrombus as well as fine structure of the aggregated platelets (Fig 4C) to arrest the bleeding. It is highly anticipated that further application will clarify not only thrombus formation but also the following fibrinogenolysis and blood vessel repair, leading to a goal of biomedical microscopy for further clinical investigation such as antithrombotic treatment under "live" CLEM imaging.

We demonstrate step-by-step procedures from "live" to "TEM" imaging of thrombus formation in vivo by newly devised "Vascular mapping" method. 


\section{References:}

[1] S Nishimura et al, Blood 119 (2012) p45-56.

[2] MJ Kuijpers and JW Heemsker, Methods Mol Biol 788 (2012), p3-19.

[3] MM Kamocka et al, J Biomed Opt 15 (2010), p016020. doi: 10.1117/1.3322676.

[4] A Sawaguchi et al, J Microsc 234 (2009), p113-7.
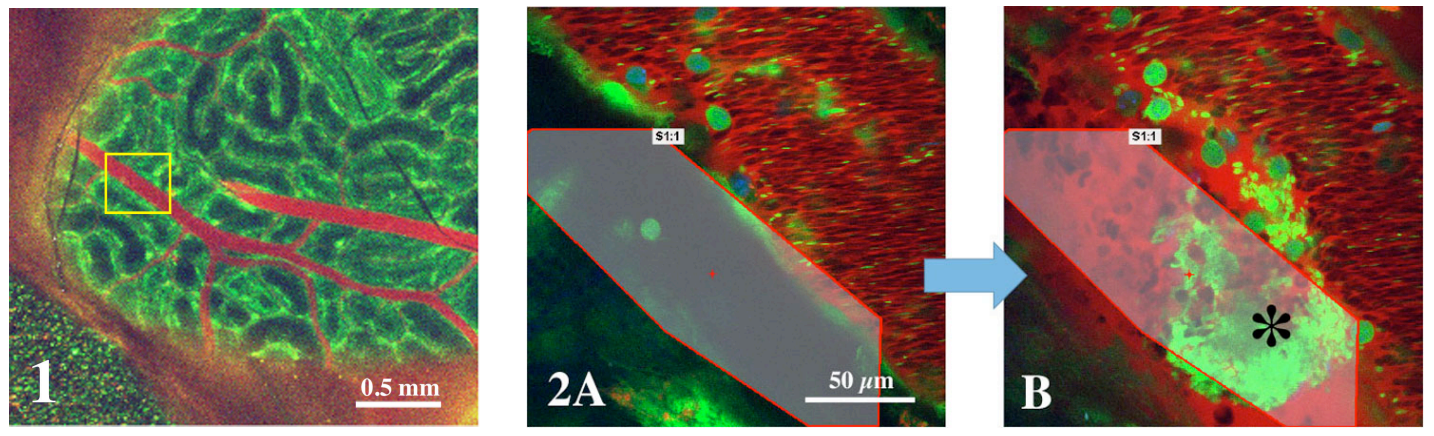

Figure 1. Representative vascular map on the mouse testicular surface vein. Boxed region corresponds to Fig 2.

Figure 2. Sequential images of laser irradiation (framed region), and thrombus formation (asterisk in B) in the boxed region of Fig 1.
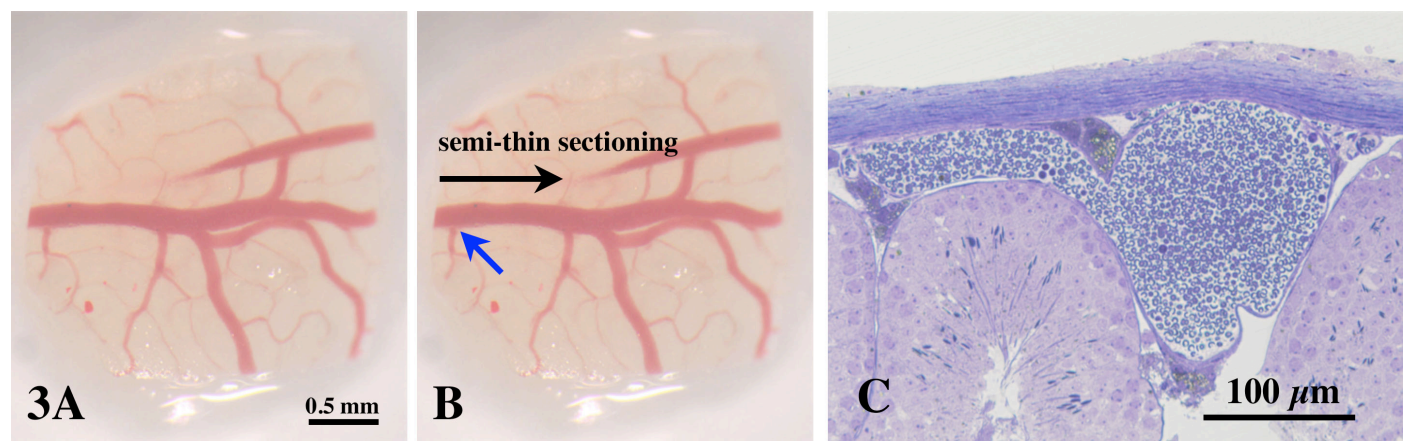

Figure 3. Excised piece of the testis (compare to Fig 1) and a semi-thin section showing the branch indicated with blue arrow in B.
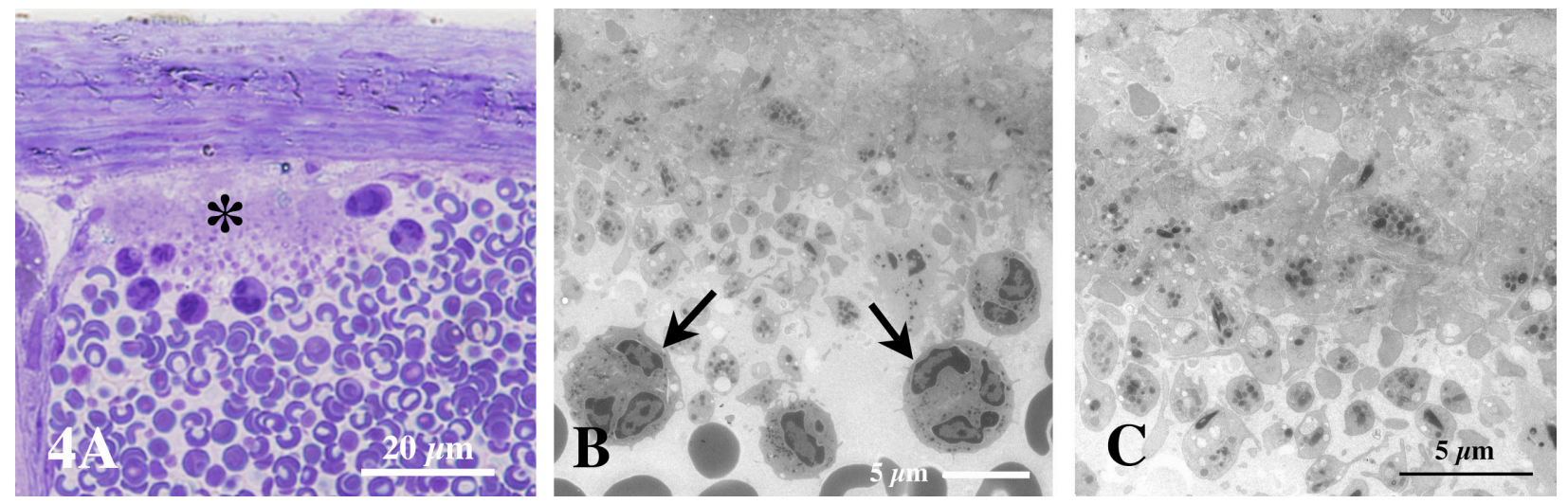

Figure 4. Semi-thin section light microscopic image of the thrombus (asterisk in A), and TEM image (B and C) obtained by re-embedding of the semi-thin section. Arrow indicate attached neutrophils. 